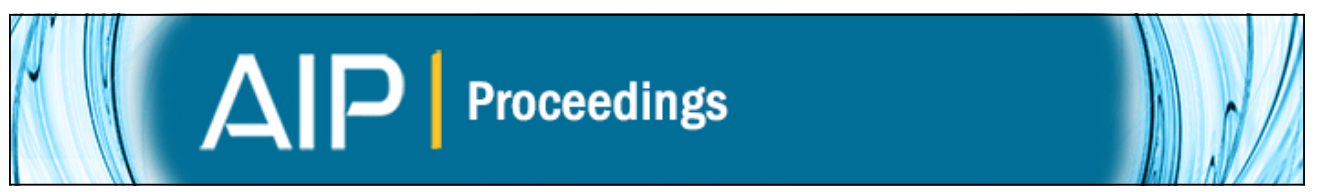

\title{
Preparation For Laser Wakefield Experiments Driven by the Texas Petawatt Laser System
}

S. A. Reed, S. Kalmykov, E. Gaul, M. Martinez, W. Henderson, P. Dong, X. Gao, J. C. Sanders, X. Wang, G. Shvets, T. Ditmire, and M. Downer

Citation: AIP Conference Proceedings 1086, 177 (2009); doi: 10.1063/1.3080901

View online: http://dx.doi.org/10.1063/1.3080901

View Table of Contents:

http://scitation.aip.org/content/aip/proceeding/aipcp/1086?ver=pdfcov

Published by the AIP Publishing

\section{Articles you may be interested in}

The Texas petawatt laser and current experiments

AIP Conf. Proc. 1507, 874 (2012); 10.1063/1.4773814

Modeling laser wakefield accelerator experiments with ultrafast particle-in-cell simulations in boosted framesa)

Phys. Plasmas 17, 056705 (2010); 10.1063/1.3358139

Wakefield driven by Gaussian $(1,0)$ mode laser pulse and laser-plasma electron acceleration Appl. Phys. Lett. 95, 091501 (2009); 10.1063/1.3187221

Snapshots of Laser-Generated Wakefields

AIP Conf. Proc. 877, 22 (2006); 10.1063/1.2409117

Limits on laser wakefield accelerators

Rev. Sci. Instrum. 70, 1983 (1999); 10.1063/1.1149698 


\title{
Preparation For Laser Wakefield Experiments Driven by the Texas Petawatt Laser System
}

\author{
S. A. Reed, S. Kalmykov, E. Gaul, M. Martinez, W. Henderson, P. Dong, \\ X. Gao, J. C. Sanders, X. Wang, G. Shvets, T. Ditmire, and M. Downer \\ Department of Physics and Institute for Fusion Studies, The University of Texas at Austin, \\ Austin, Texas 78712
}

\begin{abstract}
Laboratories around the world are planning petawatt laser driven experiments. The Texas petawatt laser offers the ability to demonstrate laser wake field acceleration (LWFA) in a unique regime with pulse duration $(\sim 160 \mathrm{fs})$ shorter than other petawatt scale systems currently in operation or under development. By focusing the $1.25 \mathrm{PW}, 200 \mathrm{~J}, 160 \mathrm{fs}$ pulses to peak intensity $\sim 10^{19} \mathrm{~W} / \mathrm{cm}^{2}$, multi-GeV electron bunches can be produced from a low density He gas jet. The rarefied plasma density $\left(5 \times 10^{16}-10^{17} \mathrm{~cm}^{-3}\right)$ required for near-resonant LWFA minimizes plasma lensing and offers long dephasing length for electron acceleration over distances $(\sim 10 \mathrm{~cm})$ exceeding the Rayleigh range. Because of the high power, the laser can be focused to a spot $\left(r_{0} \sim 100\right.$ microns) greater than the plasma wavelength $\left(r_{0}>\lambda_{p}\right)$, thus minimizing radial propagation effects. Together these properties enable the laser pulse to selfguide without the use of a preformed channel lending simplicity and stability to the overall acceleration process. Particle-in-cell (PIC) simulations show the laser experiences self-focusing which, because of ultrashort pulse duration, does not lead to a collapse of the wakefield and can generate over $3 \mathrm{GeV}$ electron energy. The presented material will include details of initial measurements of the Texas petawatt laser system, simulations of laser wakefield acceleration for the given laser parameters and the experimental setup currently under construction.
\end{abstract}

Keywords: Petawatt, Wakefield, Particle generation.

PACS: 52.38.Kd, 52.35.Mw, 52.38.Ph.

\section{INTRODUCTION}

In their introduction of the concept of laser wakefield acceleration in 1979 [1]. Tajima and Dawson showed that lasers could be compact particle accelerators in a plasma with high field gradients. In one form of laser wakefield acceleration (LWFA) a pulse of duration, shorter than a plasma period drives an electron plasma wave, while electrons to be accelerated are either externally injected or self-inject from free electrons in the plasma itself through wave breaking. However, in order to achieve such conditions laser intensities on the order of $10^{18} \mathrm{~W} / \mathrm{cm}^{2}$ are required. For typical short pulse laser systems this is possible only through tight laser focusing to a spot size $r_{0}$ of the order of $10 \mu \mathrm{m}$. This corresponds to a Rayleigh range on the order of several millimeters, which limits the expected electron energy gain. Additionally, tight laser focusing introduces strong radial fields and 3-D effects resulting in ineffective trapping and acceleration [2]. 


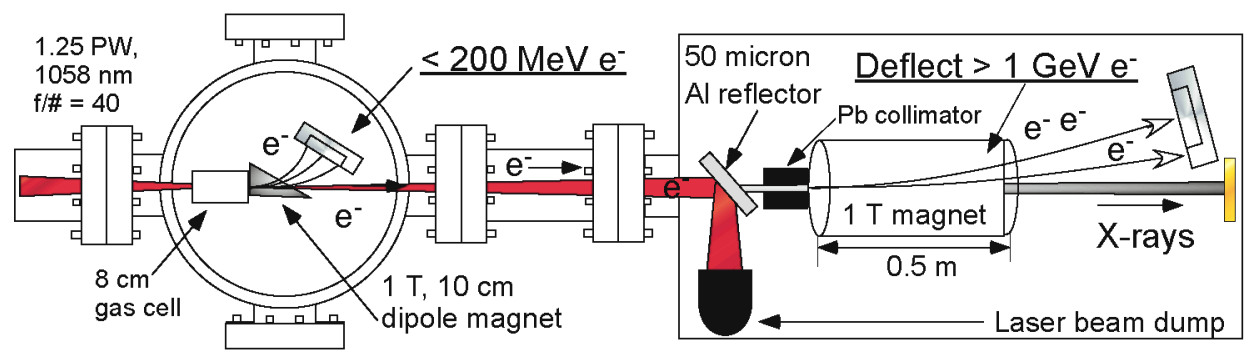

FIGURE 1. Schematic of the Texas Petawatt experimental setup.

Recent developments in petawatt (PW) class lasers have enabled ultra-high peak power systems with ultra-short pulse durations. The Texas Petawatt (TPW) laser delivers near 200 Joules of energy in a $160 \mathrm{fs}$ pulse allowing relatively large focal spots $\left(\mathrm{r}_{0} \sim 100 \mu \mathrm{m}\right)$ while still exceeding $10^{19} \mathrm{~W} / \mathrm{cm}^{2}$ intensity. The large diameter focal spot increases the Rayleigh length to several $\mathrm{cm}$ and reduces radial effects on the accelerated electrons due to the large transverse wakefield structure. The condition for near-resonant LWFA $\left(\tau_{\mathrm{L}}<\tau_{\mathrm{p}} \equiv 2 \pi / \omega_{\mathrm{p}}\right)$ is satisfied at low plasma density $\left(\mathrm{n}_{e} \sim 10^{17} \mathrm{~cm}^{-3}\right)$, thereby minimizing uncontrolled laser-plasma instabilities, making the TPW ideal for LWFA [3].

In this paper, electron generation through laser wakefield acceleration from the interaction of a $1.25 \mathrm{PW}, 150 \mathrm{fs}$ pulse with a low density $\left(5 \times 10^{16}-10^{17} \mathrm{~cm}^{-3}\right)$ gas target is studied using the PIC code WAKE in anticipation of the upcoming experiment. It is shown that the laser pulse experiences self-guiding over several Rayleigh lengths $(\sim 10$ $\mathrm{cm}$ ) which the enables generation of multi-GeV electrons without external optical guiding.

\section{EXPERIMENTAL SETUP}

The experiment will be performed with the Texas petawatt (TPW) laser system which has achieved $\sim 10^{19} \mathrm{~W} / \mathrm{cm}^{2}$ intensity with a $160 \mathrm{fs}$ pulse duration and a spot size $\mathrm{r}_{0} \sim 100 \mu \mathrm{m}$. Pulses of $100 \mathrm{fs}$ duration, $1.058 \mu \mathrm{m}$ center wavelength are generated in a tunable Ti:S oscillator, and are then stretched to $\sim 2 \mathrm{~ns}$ duration after isolation. After a Pockels cell selects pulses at $10 \mathrm{~Hz}$ repetition rate, they are parametrically amplified [4] in three stages to pulse energies near $1 \mathrm{~J}$. Then a mixed-glass power amplifier consisting of a silicate glass rod and a phosphate glass disk salvaged from the NOVA project, designed to maintain broad bandwidth and enable short pulse generation, boosts the laser energy to about $230 \mathrm{~J}$ before compression.

For the LWFA experiment the 200 Joule, $1058 \mathrm{~nm}$, laser pulse will be focused by an $\mathrm{f} / 40$ spherical mirror to a spot size of $\mathbf{r}_{0} \sim 80 \mu \mathrm{m}$. The focused laser will 


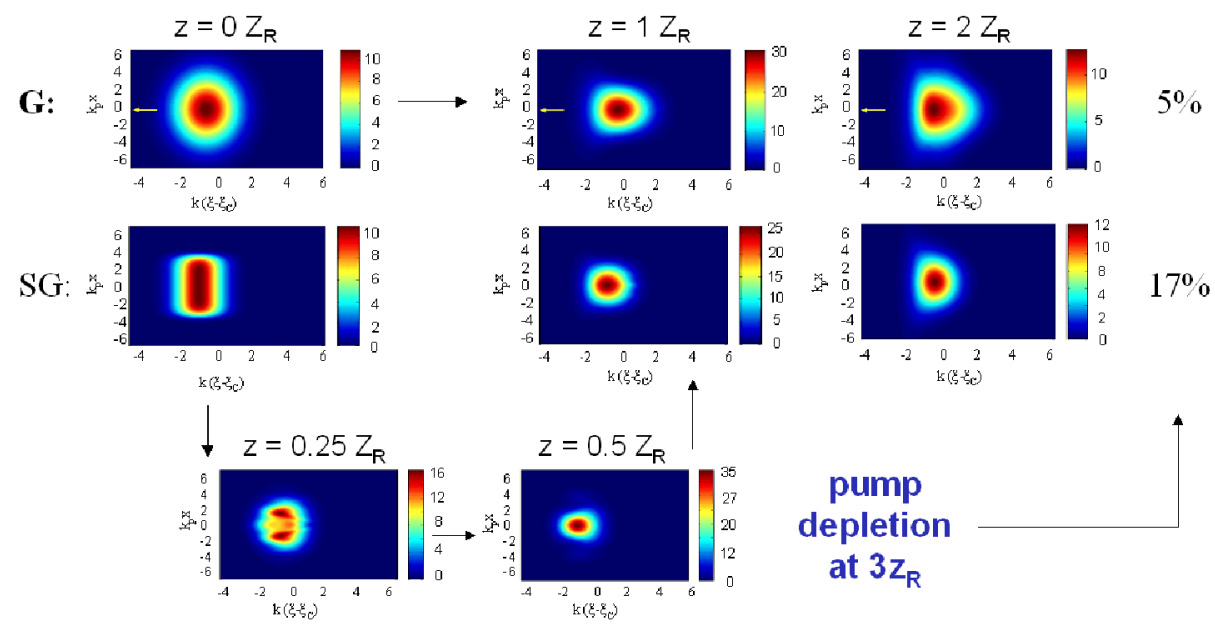

FIGURE 2. The laser fields at the entrance of the gas jet (left), at $1 / 4,1 / 2,1$, and $2 Z_{R}$ as marked. Top row depicts pulse evolution for an incident Gaussian (G) pulse profile, the bottom row for an incident super-Gaussian (SG) profile.

enter a differentially pumped gas cell containing several Torr of He that is adjustable in length up to $\sim 10 \mathrm{~cm}$. The high peak power combined with low plasma density yields peak power $\mathrm{P} \sim 7 \mathrm{P}_{\text {cr }}$, where $\mathrm{P}_{\text {cr }}$ is the critical power for relativistic selffocusing, allowing the laser to selfguide over at least 3 Rayleigh lengths.

Two tandem on-axis magnetic spectrometers measure the electron energy spectrum in two energy regimes. The first, a $10 \mathrm{~cm}$ long, $1 \mathrm{~T}$ dipole magnet deflects and measures relatively low energy electrons $(<200 \mathrm{MeV})$ thereby removing low energy particles which can interfere with characterization of the multi-GeV component. Approximately two meters after the gas cell, where transmitted laser intensity is sufficiently low that it does not create x-ray radiation, a thin Al film deflects and dumps the laser pulse while transmitting GeV-range electrons. After the beam dump a narrow channel in a $20 \mathrm{~cm}$ lead block collimates $\mathrm{GeV}$ electrons into a $0.5 \mathrm{~m}$ thick, $1 \mathrm{~T}$, cylindrical magnetic spectrometer capable of deflecting $10 \mathrm{GeV}$ electrons. The electron beam will travel approximately 8 meters to the detection phosphor screen, which is spatially separated from the on-axis $x$-ray diagnostic. A schematic of the experimental setup is shown in Fig. 1.

\section{PIC SIMULATIONS}

We simulated laser propagation and wakefield generation and acceleration for the experimental conditions described above using the fully relativistic particle-in-cell (PIC) code WAKE [5]. WAKE calculates the quasistatic plasma response to the ponderomotive force averaged over the laser period, and is implemented here in a 3D axisymmetric geometry using the extended paraxial approximation to describe 

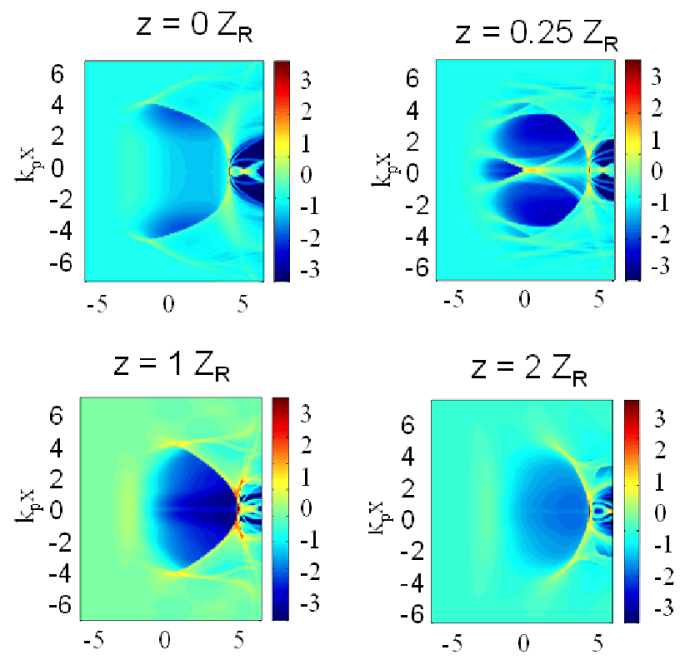

FIGURE 3. Plasma density profile at the entrance of the gas jet (left), at $1 / 4,1$, and $2 Z_{R}$ as marked for an incident super-Gaussian (SG) laser profile.

propagation of the laser pulse. In the simulations presented here the laser is linearly polarized, centered at $1058 \mathrm{~nm}$ with a laser power of $1.25 \mathrm{PW}(200 \mathrm{~J})$, a pulse duration of $150 \mathrm{fs}$ (Gaussian temporal profile) and a spot size of $80 \mu \mathrm{m}\left(\mathrm{r}_{0}\right)$. The plasma density is $10^{17} \mathrm{~cm}^{-3}$ giving 7.7 times $\mathrm{P}_{\mathrm{cr}}$. Test electrons are inserted at the $z=0$ plane (where the simulation starts), with a relativistic $\gamma$ of 4 , and an R.M.S. angular spread at injection of 0.2 radians.

Since the TPW laser does not produce an ideal Gaussian focal spot profile, a major concern is the effect of its non-ideal features on self-guiding, a critical component of the planned experiment. Accordingly, several different incident spatial profiles were simulated, such as supergaussian and Gaussian with inserted hot spots, in order to compare their self-guided propagation to that of an ideal Gaussian. Figure 2 shows the laser fields at the entrance of the gas cell (left), and at 1 , and $2 Z_{R}$ for the ideal Gaussian (G, top row) and supergaussian ( $\mathrm{SG}$, bottom row) profiles. After propagation of $\sim Z_{R}$ the profiles from the two incident pulses are essentially identical (middle column), and propagate very similarly thereafter (right-hand column). The two panels in the bottom row show details of the SG pulse propagation within the first Rayleigh length. After $1 / 4 Z_{R}$ the laser-plasma interaction begins to act as a spatial filter, quickly diffracting higher order modes leaving behind a Gaussian like structure after $Z_{R}$, at the expense of slightly higher energy loss $(17 \%)$ than for the ideal Gaussian pulse $(5 \%)$. Very similar propagation behavior was observed for a Gaussian pulse with hot spots artificially inserted into the spatial profile, showing the experiment is not sensitive to a non-ideal focal spot. The non-ideal pulse shape causes initial shedding of higher order spatial components. However, most of the pulse energy remains in the lowest order Gaussian mode which guides successfully, showing no sign of filimentation [6]. 


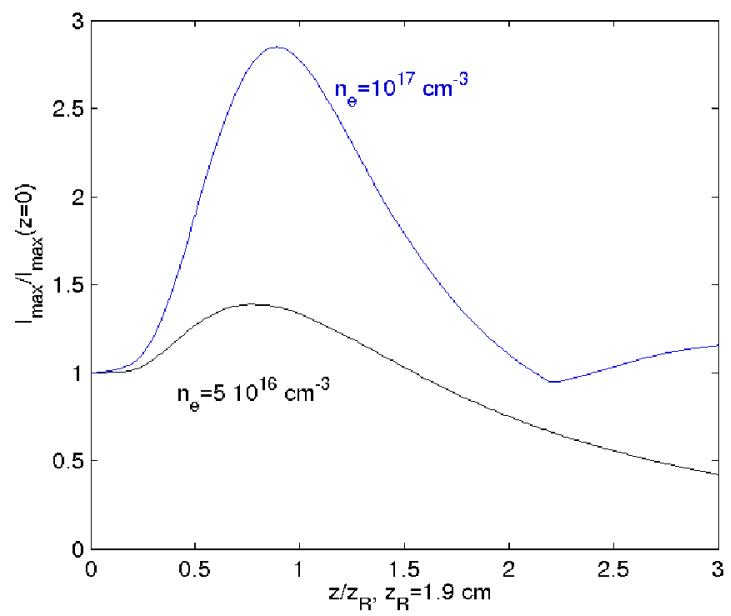

FIGURE 4. The maximum intensity of the laser pulse normalized to the input peak intensity as a function of propagation distance.

Immediately after the incident laser pulse enters the gas cell, the laser's ponderomotive force expels plasma electrons, creating a "bubble" [7]. For non-ideal supergaussian pulses strong distortions of the bubble are observed within the first half Rayleigh length as shown in Figure 3. After $\mathrm{z}^{\sim 1 / 2} Z_{\mathrm{R}}$, however, the bubble quickly recovers showing a well formed, almost completely evacuated structure throughout the rest of the plasma. The acceleration gradient is shown to change steadily and remain on the order of the cold wavebreaking limit $\mathrm{E}_{\mathrm{br}}=0.96 \mathrm{n}_{0}^{1 / 2}\left[\mathrm{~cm}^{-3}\right] \approx 30 \mathrm{GV} / \mathrm{m}$.

Figure 4 shows the maximum on-axis laser intensity as a function of propagation distance normalized to the $z=0$ peak intensity for $n_{e}$ of $5 \times 10^{16}$ and $10^{17} \mathrm{~cm}^{-3}$. The over critical pulse together with the large spatial profile allow diffraction to be compensated by relativistic self focusing. For a plasma density $10^{17} \mathrm{~cm}^{-3}$ the laser intensity increases by a factor of 3 after about one $Z_{R}$ but returns to unity, successfully guiding over $3 Z_{R}$. For the lower plasma density of $5 \times 10^{16}$ the laser's peak intensity remains stable throughout the interaction. For both density conditions the laser spectrum becomes broader and red shifted to $\sim 4$ plasma periods, but shows no significant blue shift. It is important to point out that such stable self-guiding is expected to continue past $3 Z_{R}$ which would allow for electrons to approach $10 \mathrm{GeV}$.

Figure 5 shows the electron energy and energy spread in units of gamma for 1,2 , and 3 Rayleigh lengths simulated using a Gaussian pulse profile. The energy spreads shown are directly related to the assumed initial conditions of the test electrons in the WAKE simulation, and are therefore only suggestive. Full 3D PIC simulations will be required to model self-injection accurately. However, because test electrons were injected with a wide angular and energy spread, the numerical simulation is believed to be a conservative estimate. Thus energy spread may be narrower in the actual experiment. 


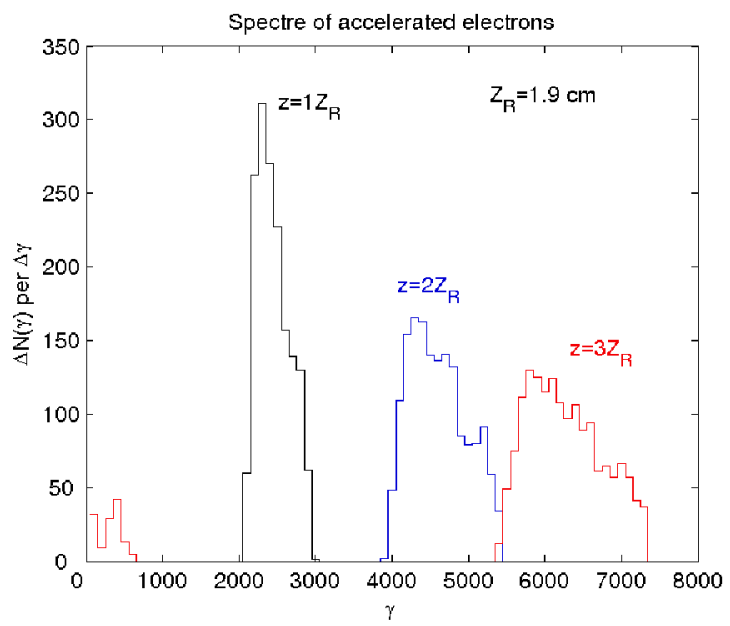

FIGURE 5. PIC simulation results showing the electron energy spectrum at 1,2 , and 3 Rayleigh lengths using a Gaussian Pulse.

\section{CONCLUSIONS}

In conclusion, the Texas Petawatt laser system has now generated 1.1 PW pulses, creating an opportunity to generate multi-GeV electrons by LWFA without external optical guiding. The high peak power and short pulse duration allow for a relatively large focal spot $\left(\mathrm{r}_{0} \sim 100 \mu \mathrm{m}\right)$, corresponding to a Rayleigh length $Z_{\mathrm{R}} \sim 3 \mathrm{~cm}$, with peak power $\mathrm{P} \sim 7 \mathrm{P}_{\text {cr. }}$. Particle-in-cell (PIC) simulations show the laser pulse selfguides over more than $3 Z_{R}(\sim 10 \mathrm{~cm})$ without beam collapse because of its ultrashort duration, and can generate at least $3 \mathrm{GeV}$ electron energy with a low energy spread. The final angular spread is less than $0.7 \mathrm{mrad}$ and the final normalized emittance of $<$ $2.5 \pi \mathrm{mm}$ mrad.

\section{ACKNOWLEDGMENTS}

This work was supported by U. S. DOE grants DEFG03-96-ER-40954, DE-FG0204ER54763, DE-FG02-04ER41321, and DE-FG02-07ER54945.

\section{REFERENCES}

1. T. Tajima and J. M. Dawson, Phys. Rev. Lett. 43, 267 (1979).

2. F. Amiranoff, S. Baton, D. Bernard, et al, Phys. Rev. Lett. 81, 995 (1998).

3. L. M. Gorbunov, S. Yu. Kalmykov, and P. Mora, Phys. Plasmas 12, 033101 (2005).

4. I. N. Ross, P. Matousek, M. Towrie, A. J. Langley, and J. L. Collier, Opt. Commun. 144, 125 (1997).

5. P. Mora and T. M. Antonsen Jr., Phys. Plasmas 4, 217 (1997). 
6. N. E. Andreev, L. M. Gorbunov, P. Mora, and R. R. Ramazashvili, Phys. Plasmas 14, 083104 (2007).

7. A. Pukhov and J. Meyer-ter Vehn, Appl. Phys. B 17, 355 (2002). 